\title{
Importância do Desenvolvimento de Fibrilação Atrial no Pós-Operatório em Cirurgia Cardíaca: Desfechos Intra-Hospitalares em Centro Terciário de Cardiologia Catarinense
}

\author{
Importance of Postoperative Atrial Fibrillation Development in Heart Surgery: \\ Intra-Hospital Outcomes in Santa Catarina Tertiary Cardiology Center
}

Ricardo Pereira da Silva ${ }^{1}$, Larissa Freitas Nunes Goldoni ${ }^{1, *}$, Kárila Scarduelli Luciano1, Ana Carolina Gern Junqueira ${ }^{2}$, Ana Carolina Caldara Barreto², Rafael De March Ronsoni ${ }^{1,2}$

ORCID IDs

Silva RP (D) https://orcid.org/0000-0002-8248-9609

Goldoni LFN (D) https://orcid.org/0000-0003-4340-0349

Luciano KS (D) https://orcid.org/0000-0002-5210-8414

\begin{abstract}
RESUMO
Objetivo: Determinar a incidência de fibrilação atrial no pós-operatório (FAPO) de cirurgia cardíaca, seu impacto sobre a morbimortalidade e o tempo de internação hospitalar em um centro terciário de cardiologia do estado de Santa Catarina, Brasil. Métodos: Estudo de coorte com 134 pacientes adultos submetidos à cirurgia cardíaca. Resultados: A incidência foi de 32,8\%. Após análise multivariada, os pacientes que não receberam betabloqueador estiveram associados à FAPO com razão de chances risco relativo (RR) 10,73 $(p<0,001)$. A maior taxa de eventos cardiovasculares (acidente vascular cerebral, mortalidade e síndrome coronariana aguda) foi de $25 \%$ no grupo FAPO vs. $10 \%$ (RR 3,21; $p=0,035$ ) o que, consequentemente, gerou tempo de internação maior nesses pacientes (19,1 vs. 12,5; $p=0,01)$. Conclusão: A incidência de FAPO foi elevada, ocasionou significativo aumento de morbimortalidade e tempo de internação hospitalar e consolidou o papel da terapia betabloqueadora na sua prevenção, podendo servir como base a futuras políticas de prevenção dessa intercorrência.
\end{abstract}

PALAVRAS-CHAVE: Fibrilação atrial; Cuidados pós-operatórios; Morbidade.

\author{
Junqueira ACG (D) https://orcid.org/0000-0001-7250-0723 \\ Barreto ACC (D) https://orcid.org/0000-0002-3359-9940 \\ Ronsoni RDM (D) https://orcid.org/0000-0001-7135-9844
}

\begin{abstract}
Objective: To determine the incidence of postoperative atrial fibrillation (PAF) of cardiac surgery, its impact on morbimortality and duration of hospital stay in a tertiary cardiology center of the state of Santa Catarina, Brazil. Methods: Cohort study with 134 adult patients submitted to cardiac surgery. Results: the incidence was $32.8 \%$. After multivariate analysis, patients who did not receive beta-blockers were associated with PAF with a relative risk odds ratio (RR) 10.73 ( $p<0.001$ ). The highest rate of cardiovascular events (cerebrovascular accident, mortality, and acute coronary syndrome) was $25 \%$ in the PAF group. $10 \%$ (RR 3.21; $p=0.035$ ) which, consequently, generated longer hospitalization time in these patients (19.1 vs. 12.5; $p=0.01$ ). Conclusion: the incidence of PAF was high, caused a significant increase in morbimortality and duration of hospital stay, and consolidated the role of beta-blocker therapy in its prevention, and may serve as a basis for future prevention policies
\end{abstract}

KEYWORDS: Atrial fibrillation; Postoperative care; Morbidity.

1. Hospital Regional Hans Dieter Schmidt - Serviço de Cardiologia - Joinville/SC- Brasil.

2.Universidade da Região de Joinville - Departamento de Medicina - Joinville/SC - Brasil.

*Autor correspondente: larissanunes_@hotmail.com

Recebido: 13 Jan 2019 | Aceito: 3 Jul 2019

Editor Associado: J Tarcisio Medeiros de Vasconcelos 


\section{INTRODUÇÃO}

A fibrilação atrial pós-operatória (FAPO) é a complicação mais prevalente após cirurgia cardíaca ${ }^{1,2}$. Estima-se que no pósoperatório (PO) de cirurgia cardíaca a incidência de FAPO varie entre 25 a $62 \%$, dependendo do tipo de cirurgia ${ }^{2,3}$. A FAPO predispõe o paciente a uma maior incidência de eventos tromboembólicos, instabilidade hemodinâmica e aumenta de $2,5$ a 4,5 vezes a chance de acidente vascular cerebral (AVC) $)^{4,5}$. O tempo de permanência hospitalar de pacientes com FAPO também é maior, gerando maior custo ao sistema de saúde ${ }^{6}$.

O pico de incidência dessa arritmia é no segundo a quarto dia PO, e estudos apontam que pode ter seu risco diminuído com a administração de drogas específicas no perioperatório ${ }^{7,8}$. A identificação de pacientes com alto risco para desenvolver FAPO pode ser de grande valia para intervenção precoce e medidas de prevenção para diminuição de morbimortalidade 5 . Embora alguns fatores, como idade avançada e comorbidades subjacentes, já sejam estabelecidos para o desenvolvimento dessa taquiarritmia, os mecanismos fisiopatológicos ainda não são completamente elucidados, mas a ativação do sistema nervoso simpático e o processo inflamatório causado pela cirurgia são os mais aceitos ${ }^{1,9-11}$.

Embora essas taquiarritmias sejam consideradas transitórias e benignas, em determinados perfis clínicos de pacientes podem ter diferentes desfechos ${ }^{1,4}$. Apesar dos esforços para diminuir sua ocorrência, o aprimoramento da técnica operatória e dos métodos de anestesia não causou redução significativa nessa complicação e sua prevenção ainda é um desafio ${ }^{3,4}$.

O objetivo deste trabalho é avaliar os desfechos intrahospitalares associados ao desenvolvimento de FAPO. Além disso, determinar a incidência e seus fatores associados em um centro terciário de cardiologia catarinense.

\section{MÉTODOS}

Estudo de coorte histórica retrospectiva com 147 pacientes adultos (idade $\geq 18$ anos) submetidos à cirurgia cardíaca (com ou sem circulação extracorpórea, devido à cardiopatia isquêmica e/ou valvar associada, ou não, a outro procedimento) em 2015 no Hospital Regional Hans Dieter Schmidt, em Joinville, estado de Santa Catarina. Desses, excluíram-se 13pacientes que tinham diagnóstico de fibrilação atrial (FA) paroxística, persistente ou permanente, restando 135 pacientes para análise. Os desfechos estudados foram a ocorrência da FAPO, clínicos [mortalidade, tempo de internação, eventos cardiovasculares - síndrome coronariana aguda (SCA) e AVC] e fatores associados ao desenvolvimento de FAPO.

Conforme protocolo institucional, todos os pacientes foram submetidos à monitorização cardíaca contínua por 48 horas em ambiente de terapia intensiva e por exames eletrocardiográficos diários até a alta hospitalar. Eletrocardiogramas adicionais foram realizados em caso de sintomas cardiovasculares e/ou a presença de taquicardia assintomática.

FA foi definida como arritmia supraventricular cujo traçado eletrocardiográfico apresentou ondas "f" de morfologia e amplitude variáveis, com ritmo ventricular irregular. Foram considerados para o trabalho episódios de FA com duração mínima de 30 segundos ou que necessitaram de tratamento devido à sintomatologia ou instabilidade hemodinâmica.

Os parâmetros ecocardiográficos de fração de ejeção do ventrículo esquerdo (FEVE) e diâmetro de átrio esquerdo (AE) foram coletados pelo mais recente exame encontrado nos registros, o que inclui tanto exames transesofágicos (ETEs) como transtorácicos (ETTs). A fração de ejeção foi calculada utilizando os métodos de Teicholz ou Simpson, de acordo com a presença de disfunção segmentar. Os diâmetros do átrio foram avaliados utilizando o modo $\mathrm{M}$.

Os prontuários foram revisados com o intuito de registrar as informações clínicas necessárias para preenchimento do protocolo clínico. Os referidos diagnósticos clínicos foram definidos de acordo com publicações prévias ${ }^{12}$.

Mediu-se a força de associação de cada um dos fatores para FAPO por meio de estimativas de risco relativo (RR) com intervalos de confiança de 95\% (IC95\%). A análise de dados foi realizada com o auxílio do programa SPSS versão 8.0. Na análise multivariada, utilizou-se a regressão logística binária e como medida de associação a razão de chances (RC). No processo de construção do modelo verificou-se a importância de cada componente por meio do teste da razão de verossimilhança. Foi usado o valor de -2log verossimilhança (deviance), medida para determinar quão bem o modelo se ajusta aos dados. As estimatiaas por intervalo foram calculadas com IC95\%. Todas as variáveis com $\mathrm{p}<0,25$ (análise univariada) foram candidatas a entrar no modelo, de acordo com a metodologia de Hosmer e Lemeshow ${ }^{13}$. Permaneceram no modelo apenas as variáveis com valor de $\mathrm{p}<0,05$.

O comitê de ética em pesquisa do hospital aprovou o protocolo do estudo (no. 1.779.036) 


\section{RESULTADOS}

Dentre os 134 pacientes submetidos à cirurgia cardíaca, 32,8\% desenvolveram FAPO. Essa incidência está relacionada ao tempo de $\mathrm{PO}$ e mostrou que cerca de $80 \%$ ocorreram do segundo ao quarto dia de $\mathrm{PO}$, sendo o pico no terceiro dia (40\%).

Quanto ao perfil clínico dos pacientes, os únicos que demonstraram significância, em análise bivariada, foram a idade superior a 70 anos $(p=0,003)$ e a não utilização de terapia betabloqueadora no período perioperatório $(\mathrm{p}<0,001)$ (Tabela 1$)$.

$\mathrm{Na}$ Tabela 2, após análise multivariada, a não utilização de terapia de betabloqueadora permaneceu estatisticamente significativa com RC de 10,73 (IC95\% $3,47-33,12 ; \mathrm{p}<0,001)$.

A mortalidade geral na amostra foi de $17 \%$ e houve tendência de maior mortalidade nos pacientes de FAPO $(0,19 \pm 0,39$ vs. $0,07 \pm 0,25 ; p>0,05)$ (Fig. $1)$, mas quando se agrupam em desfechos compostos, observam-se eventos cardiovasculares (AVC, mortalidade e SCA) com incidência de 25\% no grupo que desenvolveu FAPO vs. 10\% [RC 3,21; IC95\% 1,08-11,58; $\mathrm{p}=0,035$ ] (Tabela 3).

O tempo de internação mostrou-se maior nos pacientes com essa complicação $(19,11 \pm 15,87$ vs. $12,58 \pm 12, \mathrm{p}=0,01)$ (Fig. 2).

Tabela 1. Análise bivariada de variáveis categóricas e fibrilação atrial no pós-operatório (FAPO).

\begin{tabular}{|c|c|c|c|c|}
\hline Período & Variável & $\begin{array}{c}\text { FAPO } \\
n=44(\%)\end{array}$ & $\begin{array}{c}\text { Sem FAPO } \\
n=90(\%)\end{array}$ & p-valor \\
\hline \multirow[t]{14}{*}{ Pré-operatório } & Sexo masculino & $28(63,6)$ & $56(62,2)$ & 0,874 \\
\hline & Idade superior a 70 anos & $14(31,8)$ & $10(11,1)$ & $0,003^{+}$ \\
\hline & Doença pulmonar crônica obstrutiva & $19(43,2)$ & $29(32,2)$ & 0,214 \\
\hline & Clearance de creatinina $<50 \mathrm{mg} / \mathrm{dL}$ & $05(11,4)$ & $04(4,40)$ & $0,154 *$ \\
\hline & Arteriosclerose extracardíaca & $08(18,2)$ & $19(21,1)$ & 0,691 \\
\hline & Cirurgia cardíaca prévia & $01(2,30)$ & $05(5,60)$ & $0,663 *$ \\
\hline & Endocardite & $03(6,80)$ & $03(3,30)$ & $0,394 *$ \\
\hline & Estado crítico no pré-operatório & $06(13,6)$ & $07(7,80)$ & $0,353 *$ \\
\hline & Angina de repouso & $02(4,50)$ & $06(6,70)$ & $1,000 *$ \\
\hline & Fração de ejeção < 50\% & $15(32,6)$ & $29(33,0)$ & 0,968 \\
\hline & Infarto agudo recente & $10(22,7)$ & $29(32,2)$ & 0,256 \\
\hline & Hipertensão arterial pulmonar & $06(13,6)$ & $04(4,40)$ & $0,080 *$ \\
\hline & Cirurgia de urgência & $03(6,80)$ & $04(4,40)$ & $0,683 *$ \\
\hline & Doença mitral importante & $05(11,4)$ & $10(11,1)$ & $1,000 *$ \\
\hline \multirow[t]{3}{*}{ Transoperatório } & Cirurgia de revascularização miocárdica & $31(70,5)$ & $69(76,7)$ & 0,438 \\
\hline & Cirurgia valvar & $11(25,0)$ & $19(18,9)$ & 0,414 \\
\hline & Cirurgia combinada & $02(4,50)$ & $04(4,40)$ & $1,000 *$ \\
\hline \multirow[t]{4}{*}{ Pós-operatório (PO) } & Balanço hídrico > 1.500 mL em 48 horas PO & $33(75,0)$ & $54(60,0)$ & 0,087 \\
\hline & Uso de anti-inflamatórios não esteroides & $02(4,50)$ & $02(2,20)$ & $0,59 *$ \\
\hline & Sem inibidores enzima conversora da angiotensina no pré- e PO & $27(61,4)$ & $44(48,9)$ & 0,174 \\
\hline & Sem Beta-bloqueador no pré- e PO & $40(90,9)$ & $46(51,1)$ & $0,000^{+}$ \\
\hline
\end{tabular}

Método estatístico empregado: teste qui-quadrado e, quando necessário, teste exato de Fischer*. ${ }^{\dagger} p<0,05$ considerado estatisticamente significativo.

Tabela 2. Variáveis associadas à fibrilação atrial no pós-operatório (FAPO) na regressão logística (análise univariada e multivariada com e sem o desfecho FAPO).

\begin{tabular}{ccccc}
\hline Variável & RC bruto (IC95\%) & p-valor & RC ajustado (IC95\%) & p-valor \\
\hline Sem Beta-bloqueador pré- e PO & $8,445(2,608-27,346)$ & 0,000 & $10,735(3,479-33,125)$ & $0,000^{\dagger}$ \\
Idade $>$ 70 anos & $2,127(0,775-5,837)$ & 0,115 & - & - \\
\hline
\end{tabular}

Método estatístico empregado: regressão logística binária univariada e multivariada; IC95\% = intervalo de confiança de 95\%; PO = pós-operatório; $\mathrm{RC}=$ razão de chances. ${ }^{\dagger}$ Variáveis que permaneceram no modelo final $p<0,05$. 


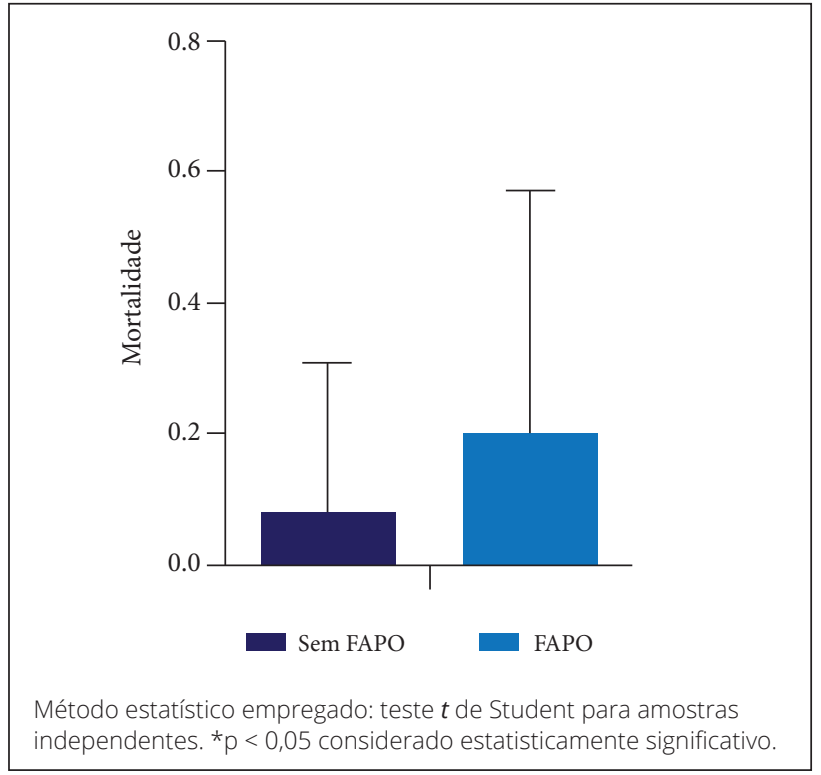

Figura 1. Mortalidade com e sem fibrilação atrial no pósoperatório (FAPO).

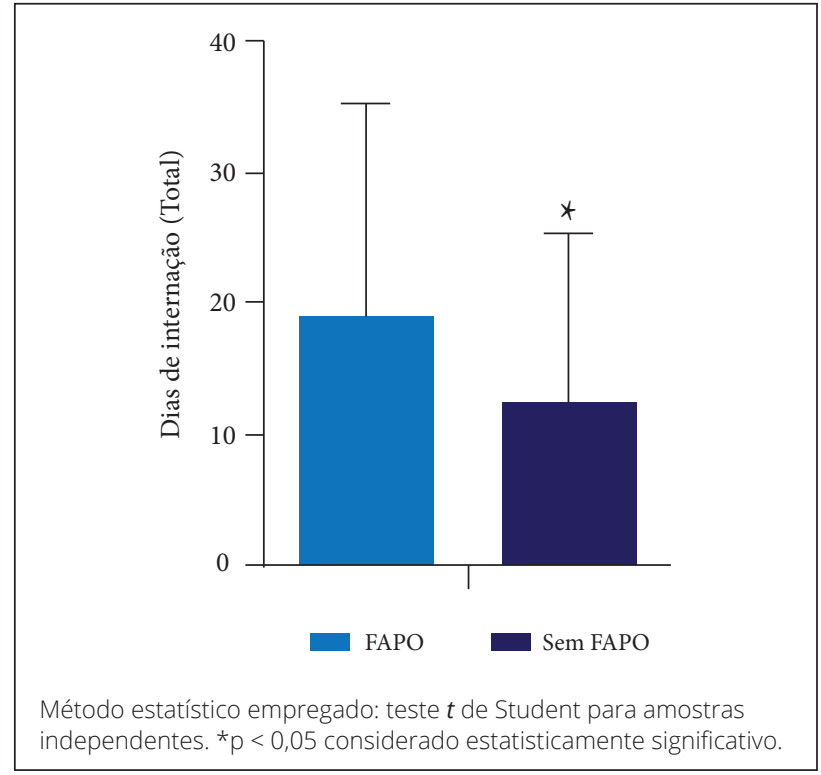

Figura 2. Dias de internação total com e sem fibrilação atrial no pós-operatório (FAPO).

Tabela 3. Análise de eventos cardiovasculares.

\begin{tabular}{ccccc}
\hline Variável & $\begin{array}{c}\text { FAPO } \\
\mathrm{n}=\mathbf{4 4}(\%)\end{array}$ & $\begin{array}{c}\text { Sem FAPO } \\
\mathrm{n}=\mathbf{9 0}(\%)\end{array}$ & $\begin{array}{c}\text { RC ajustado } \\
\text { (IC95\%) }\end{array}$ & p-valor \\
\hline $\begin{array}{c}\text { Eventos } \\
\text { cardiovasculares }\end{array}$ & $11(25 \%)$ & $9(10)$ & $3,21(1,08-11,58)$ & $0,035^{\dagger}$ \\
\hline
\end{tabular}

FAPO = fibrilação atrial no pós-operatório; IC95\% = intervalo de confiança de 95\%; RC = razão de chances. Método estatístico empregado: teste qui-quadrado e, quando necessário, o teste exato de Fischer, ${ }^{\dagger} \mathrm{p}<0,05$ considerado estatisticamente significativo.

\section{DISCUSSÃO}

Apesar da evolução das técnicas cirúrgicas e do manejo PO, a FAPO continua sendo uma complicação muito frequente ${ }^{14-17}$. Encontrou-se incidência de 32,8\%, índice semelhante à média relatada em outros trabalhos publicados ${ }^{18-20}$. Vale relatar que se utilizou monitorização contínua no período de terapia intensiva e após eletrocardiograma intermitente, fato que pode ter subestimado FAPO de caráter assintomático. Alguns estudos nos quais foi utilizada monitorização contínua, as taxas atingiram 44 a 64\%; todavia essa modalidade é adotada em uma minoria de trabalhos publicados ${ }^{21,22}$. Os dados demonstram, portanto, incidência elevada, estimulando a busca de fatores que contribuam para a FAPO, sendo assim um dos objetivos deste estudo.

A cronologia foi compatível com dados já publicados, nos quais a maior parte dos episódios ocorre até o quarto dia de PO; neste estudo ocorreu em $80 \%$ dos casos ${ }^{23}$.

Antes de citar os fatores associados à FAPO, é importante estabelecer os mecanismos multifatoriais associados ao desenvolvimento da arritmia ${ }^{24}$. Dentre eles, talvez o mais facilmente abordado por medidas profiláticas seja o desequilíbrio autonômico, em muito ocasionado pela hiperestimulação simpática, por meio da utilização de medicações betabloqueadoras. Neste estudo, realmente se detectou que a não utilização dessa classe medicamentosa agregou maior impacto na ocorrência da arritmia. Após análise multivariada, obteve-se RC de 10,73, refletindo a fundamental importância do bloqueio do sistema nervoso simpático nesse contexto clínico. $\mathrm{E}$ isso é confirmado na literatura por uma meta-análise com 4.074 pacientes que encontrou RR de 0,35 (IC95\% 0,26-0,49) associado a esse achado protetor com a utilização da terapia ${ }^{25}$. Importante ressaltar que o pior cenário clínico descrito seria a não utilização no pré- e PO da medicação ${ }^{25,26}$. Esses resultados solidificam esse fator como plenamente modificável por meio da conscientização médica de sua utilização nesse período crítico. Associado a isso, poderiam ser utilizados como orientação ou indicadores para políticas de prevenção dessa complicação. 
Idade superior 70 anos é um preditor de FAPO importante na literatura. Mathew et al. ${ }^{27}$ documentaram que, para cada década, há aumento de $75 \%$ na chance da ocorrência arrítmica. Os pesquisadores concluíram que, com base na idade por si só, qualquer pessoa com idade superior a 70 anos é considerada de alto risco para desenvolver $\mathrm{FAPO}^{27}$. Neste estudo houve associação em análise bivariada, mas após regressão logística a idade não permaneceu significativamente estatística, muito provavelmente relacionada ao tamanho da amostra.

Com relação aos desfechos clínicos, a mortalidade total é associada ao desenvolvimento de FAPO. Quando se utiliza a base de dados da Society of Thoracic Surgeons (STS), atingindo 49.264 pacientes, o grupo que desenvolveu FAPO tem o dobro de chance de mortalidade (RR 2,04, $\mathrm{p}<0,001)^{28}$. Confirmando os dados da STS, Almassi et al. ${ }^{29}$,em análise de 3.855 pacientes submetidos à cirurgia cardíaca, encontraram mortalidade intra-hospitalar de 6\% no grupo de pacientes com FAPO vs. 3\% no grupo sem arritmia. Neste estudo verificou-se tendência de aumento com FAPO, mas com ausência de significância estatística, o que poderia ser explicado pela associação do tamanho da amostra (reduzido) e da incidência de mortalidade (aumentada) quando comparado com estudos já publicados.

A FAPO está associada à incidência aumentada de eventos mórbidos importantes ${ }^{29}$, tal como aumento do risco tromboembólico ${ }^{30}$ e isquemia miocárdica, e isso é particularmente pronunciado no aumento de AVC, tal como analisado na base de dados da STS, entre 2004 e 2005, de cirurgias de revascularização miocárdica, em que se verificou aumento da prevalência de AVC (4 vs. $1.9 \%, \mathrm{p}=0.002)^{6}$. Neste estudo, agruparam-se em desfecho combinado de mortalidade AVC e SCA e atingiu-se RC de 3,2 ( $<<0,05)$, o que demonstra o potencial mórbido da FAPO.

Diante dos dados encontrados de maior morbidade associada à FAPO, é compreensível esperar um aumento do tempo de internação em dias, como observado em nosso estudo $(19,11 \pm 15,87$ vs. $12,58 \pm 12 ; \mathrm{p}=0,01)$. Dados de um grupo brasileiro demonstraram resultados semelhantes de maior tempo de internação em dias $(16,9 \pm 12,3$ vs. $9,2 \pm 4, \mathrm{p}<0,001)^{31}$.

Importante ressaltar que como a origem dos dados é de banco de dados clínico, têm-se estimativa do "mundo real" e de dados do sistema público brasileiro. Isso consolida a políticas futuras de prevenção de FAPO para minimizar efeitos clínicos e financeiros aos sistemas de saúde.

\section{CONTRIBUIÇÃO DOS AUTORES}

Conceitualização, Silva R e Ronsoni R e Goldoni L; Metodologia, Silva Re Ronsoni Re Scarduelli K, Investigação, Barreto ACC e Junqueira ACG; Redação - primeira versão, Silva Re Barreto ACC e Junqueira ACG; Redação - Revisão e Edição, Goldoni L e Ronsoni R; Supervisão Ronsoni R e Scarduelli K.

\section{REFERÊNCIAS}

1. Shen J, Lall S, Zheng V, Buckley P, Damiano RJ, Schuessler RB. The persistent problem of new onset postoperative atrial fibrillation: a single institution experience over two decades. The J Thorac Cardiovasc Surgery. 2011;141(2):55970. https://doi.org/10.1016/j.jtcvs.2010.03.011

2. Villareal RP, Hariharam R, Liu BC, Kar B, Lee W, Elayda M, et al. Postoperative atrial fibrillation and mortality after coronary artery bypass surgery. J Am Coll Cardiol. 2004;43(5):742-8. https://doi.org/10.1016/j.jacc.2003.11.023

3. Ommen SR, Odell JA, Marshall S. Stanton. Atrial arrhythmias after cardiothoracicsurgery. N EnglJ Med. 1997;336(20):142934. https://doi.org/10.1056/NEJM199705153362006

4. Mariscalco G, Klersy C, Zanobini M, Banach M, Ferrarese $\mathrm{S}$, Borsani $\mathrm{P}$, et al. Atrial fibrillation after isolates coronary surgery affects late survival.Circulation. 2018;118(16):16128. https://doi.org/ 10.1161/CIRCULATIONAHA.108.777789
5. Geovanini GR, Alves RJ, Brito G de, Miguel GAS, Glauser VA, Nakiri K. Fibrilação atrial no pós-operatório de cirurgia cardíaca: quem deve receber quimioprofilaxia? Arq Bras Cardiol. 2009;92(4):326-30. https://doi.org/10.1590/S0066$782 \times 2009000400013$

6. Steinberg BA, Zhao Y, He X, Hernandez AF, Fullerton DA, Thomas $\mathrm{KL}$, et al. Management of postoperative atrial fibrillation and subsequent outcomes in contemporary patients undergoing cardiac surgery: insights from the Society of Thoracic Surgeons CAPS-Care Atrial Fibrillation Registry. Clin Cardiol. 2014;37(1):7-13. https://doi. org/10.1002/clc.22230

7. Mahoney EM, Thompson TD, Veledar E, Williams J, Weintraub WS. Cost-effectiveness of targeting patients undergoing cardiac surgery for therapy with intravenous amiodarone to prevent atrial fibrillation. J Am Coll Cardiol. 2002;40(4):73745. https://doi.org/10.1016/S0735-1097(02)02003-X 
8. Silva RG da, Lima GG de, Guerra N, Bigolin AV, Petersen LC. Proposta de escore de risco para predição de fibrilação atrial após cirurgia cardíaca. Rev Bras Cir Cardiovasc. 2010;25(2):18319. https://doi.org/10.1590/S0102-76382010000200009

9. Creswell LL, Schuessler RB, Rosenbloom M, Cox JL. Hazards of postoperative atrial arrhythmias. Ann Thoruc Surg. 1993;56(3):539-49. https://doi.org/10.1016/00034975(93)90894-n

10. Angelini P, Feldman MI, Lufschanowski R, Leachman RD. Cardiac arrhythmias during and after heart surgery: diagnosis and management. Prog Cardiovasc Dis. 1974;16(5):469-95. https://doi.org/10.1016/0033-0620(74)90007-3

11. Albahrani MJ, Swaminathan M, Phillips-Bute B, Smith PK, Newman MF, Mathew JP, et al. Postcardiac surgery complications: association of acute renal dysfunction and atrial fibrillation. Anesth Analg. 2003;96(3):637-43. https:// doi.org/10.1213/01.ane.0000047886.81598.2c

12. Lip GY, Nieuwlaat R, Pisters R, Lane DA, Crijns HJ. Refining clinical risk stratification for predicting stroke and thromboembolism in atrial fibrillation using a novel risk factor-based approach: the Euro Heart Survey on atrial fibrillation. Chest. 2010;137(2):263-72. https://doi.org/ 10.1378/chest.09-1584

13. Hosmer DW, Lemeshow S. Applied logistic regression. New York: John Wiley; 1989.

14. Mathew JP, Parks R, Savino JS, Friedman AS, Koch C, Mangano DT, et al. Atrial fibrillation following coronary artery bypass graft surgery: predictors, outcomes, and resource utilization. MultiCenter Study of Perioperative Ischemia Research Group. JAMA. 276(4):300-6. https://doi. org/10.1001/jama.1996.03540040044031

15. Echahidi N, Mohty D, Pibarot P, Després J-P, O'Hara G, Champagne J, et al. Obesity and metabolic syndrome are independent risk factors for atrial fibrillation after coronary artery bypass graft surgery. Circulation. 2007;116(11 Suppl):1213-9. https://doi.org/10.1161/ CIRCULATIONAHA.106.681304

16. Osranek M, Fatema K, Qaddoura F, Al-Saileek A, Barnes ME, Bailey KR, et al. Left atrial volume predicts the risk of atrial fibrillation after cardiac surgery: a prospective study. J Am Coll Cardiol. 2006;48(4):779-86. https://doi.org/10.1016/j. jacc.2006.03.054

17. Sabzi F, Zokaei AH, Moloudi AR. Predictors of atrial fibrillation following coronary artery bypass grafting. Clin Med Insights Cardiol. 2011;5:67-75. https://doi.org/10.4137\%2FCMC. $\mathrm{S} 7170$

18. Echahidi N, Pibarot P, O'Hara G, Mathieu P. Mechanisms, prevention, and treatment of atrial fibrillation after cardiac surgery. J Am Coll Cardiol. 2008;51(8):793-801. https://doi. org/10.1016/j.jacc.2007.10.043.

19. Maisel WH, Rawn JD, Stevenson WG. Atrial fibrillation after cardiac surgery. Ann Intern Med. 2001;135(12):1061. https:// doi.org/10.7326/0003-4819-135-12-200112180-00010
20. Nazeri A, Razavi M, Elayda MA, Lee V-V, Massumi A, Wilson $\mathrm{JM}$, et al. Race/ethnicity and the incidence of new-onset atrial fibrillation after isolated coronary artery bypass surgery. Hear Rhythm. 2010;7(10):1458-63. https://doi. org/10.1016/j.hrthm.2010.06.037

21. Siebert J. Atrial fibrillation after coronary artery bypass grafting without cardiopulmonary bypass. Eur J CardioThoracic Surg. 2000;17(5):520-3. https://doi.org/10.1016/ S1010-7940(00)00368-7

22. Helgadottir S, Sigurdsson MI, Ingvarsdottir IL, Amar DO, Gudbjartsson T. Atrial fibrillation following cardiac surgery: risk analysis and long-term survival. J Cardiothorac Surg. 2012;7(1):87-93. https://doi.org/10.1186/1749-8090-7-87

23. Kannel WB, Abbott RD, Savage DD, McNamara PM. Epidemiologic features of chronic atrial fibrillation. N Engl J Med. 1982;306(17):1018-22. https://doi.org/10.1056/ NEJM198204293061703

24. Yadava M, Hughey AB, Crawford TC. Postoperative atrial fibrillation: incidence, mechanisms, and clinical correlates. Cardiol Clin. 2014;32(4):627-36. https://doi.org/10.1016/j. ccl.2014.07.002

25. Crystal E, Garfinkle MS, Connolly SS, Ginger TT, Sleik K, Yusuf SS. Interventions for preventing post-operative atrial fibrillation in patients undergoing heart surgery. Cochrane Database Syst Rev. 2004;18(4):CD003611. https://doi. org/10.1002/14651858.CD003611.pub2

26. Andrews TC, Reimold SC, Berlin JA, Antman EM. Prevention of supraventricular arrhythmias after coronary artery bypass surgery. A meta-analysis of randomized control trials. Circulation. 1991;84(5 Suppl):III236-44.

27. Mathew JP, Fontes ML, Tudor IC, et al. A multicenter risk index for atrial fibrillation after cardiac surgery. JAMA. 2004;291(14):1720-9. https://doi.org/10.1001/ jama.291.14.1720

28. LaPar DJ, Speir AM, Crosby IK, et al. Postoperative atrial fibrillation significantly increases mortality, hospital readmission, and hospital costs. Ann Thorac Surg. 2014;98(2):527-33; discussion 533. https://doi. org/10.1016/j.athoracsur.2014.03.039

29. Almassi GH, Schowalter T, Nicolosi AC, Aggarwal A, Moritz TE, Henderson WG, et al. Atrial fibrillation after cardiac surgery: a major morbid event? Ann Surg. 1997;226(4):501-11. https://doi.org/10.1097/00000658-199710000-00011

30. Fuller JA, Adams GG, Buxton B. Atrial fibrillation after coronary artery bypass grafting. Is it a disorder of the elderly? J Thorac Cardiovasc Surg. 1989;97(6):821-5.

31. Silva RG, Lima GG, Laranjeira A, Costa AR, Pereira E, Rodrigues R. Fatores de risco e morbimortalidade associados à fibrilação atrial no pós-operatório de cirurgia cardíaca. Arq Bras Cardiol. 2004;83:99-104. https://doi.org/10.1590/ s0066-782X2004001400002 